\title{
Acute appendicitis due to hair follicle obstruction: a rare case report
}

\author{
Samir Abdullazade ${ }^{1}$, Bahattin Bayar², Mehmet Can³, Ezgi Altinsoy², Ilker Kiziloglu² \\ ${ }^{1}$ Pathology Laboratory, Mus State Hospital, Mus, Turkey \\ ${ }^{2}$ General Surgery Clinic, Mus State Hospital, Mus, Turkey \\ ${ }^{3}$ Paediatric Surgery Clinic, Mus State Hospital, Mus, Turkey
}

Gastroenterology Rev 2017; 12 (2): 156-158

DOI: https://doi.org/10.5114/pg.2017.68043

\begin{abstract}
Address for correspondence: Samir Abdullazade MD, Department of Pathology, Tepecik Training and Research Hospital, 35180 Izmir Turkey, phone: +90 5316308439, e-mail: abdullazade@gmail.com
\end{abstract}

Acute appendicitis is one of the most common emergency surgical cases, which usually develops secondary to an obstruction. Acute appendicitis due to a hair follicle obstruction is very rare. We present 2 unique cases of acute appendicitis with hair follicle in the lumen.

Case 1. A 27-year-old male patient was admitted to the emergency department with the complaint of abdominal pain. It was learnt from his history that his pain had started in the epigastric area 2 days previously, and then localised in the lower right quadrant. He had a lack of appetite and no nausea or vomiting. The physical examination revealed tenderness in the lower right quadrant, but there were no defence or rebound. His vital parameters were normal and the laboratory analyses revealed a white blood cell (WBC) count of 12,700 . The appendix could not be visualised in the abdominal ultrasonography (US). His modified Alvarado score was 6 , and the computerised abdominal tomography revealed that the diameter of the appendix was $8 \mathrm{~mm}$ and contamination in the surrounding mesenteric tissue (suspected in terms of acute appendicitis). The patient was taken into surgery with the pre-diagnosis of acute appendicitis. The abdomen was accessed through a McBurney incision, and intraoperatively it was observed that the appendix was inflamed and oedematous, and an appendectomy was performed. No problem was observed during the post-operative follow-up, and he was discharged on the second post-operative day.

Case 2. A 16-year-old male patient was admitted to the emergency department with the complaints of abdominal pain and bile-free vomiting. It was learnt from his history that his pain had continued for 2 days, which had been accompanied by bile-free vomiting for the last day. The patient stated that he last passed stools with normal consistency 1 day ago, and his vital parameters were normal except for increased body temperature $\left(37.7^{\circ} \mathrm{C}\right)$. A physical examination revealed significant tenderness, defence, and rebound located in the lower right quadrant. He had decreased bowel sounds, and the rectal examination revealed an empty rectum. The genital examination was normal.

The laboratory analyses revealed a WBC count of 18,400 (82\% neutrophils) and an increased level of C-reactive protein (CRP) of 11.2. His anterior/posterior chest $\mathrm{X}$-rays and plain abdominal X-ray in the standing position were normal, and US revealed a blind-ending aperistaltic non-compressed intestinal structure of $7 \mathrm{~mm}$ in diameter in the lower right quadrant of the abdomen.

The patient was taken into surgery with the diagnosis of acute appendicitis. A standard appendectomy procedure was performed through laparotomy using a Rockey-Davis incision to remove the inflamed appendix. No problem was observed during the post-operative follow-up, and he was discharged on the third post-operative day.

In the first case, the macroscopic examination revealed marked thinning of the appendiceal wall, which was consistent with perforation. Macroscopically there was hair in the lumen of the appendix in the second case. In both cases, a hair follicle was observed in the appendiceal lumen as well as acute appendicitis and periappendicitis as observed through light microscopy (Figures 1, 2).

Acute appendicitis is one of the most common emergency surgical cases, which usually develops secondary to an obstruction. Several factors are involved in the aetiology. Acute appendicitis due to a foreign body is very rare in adults when compared to children [1]. Various foreign bodies such as sewing needles, endodontic 

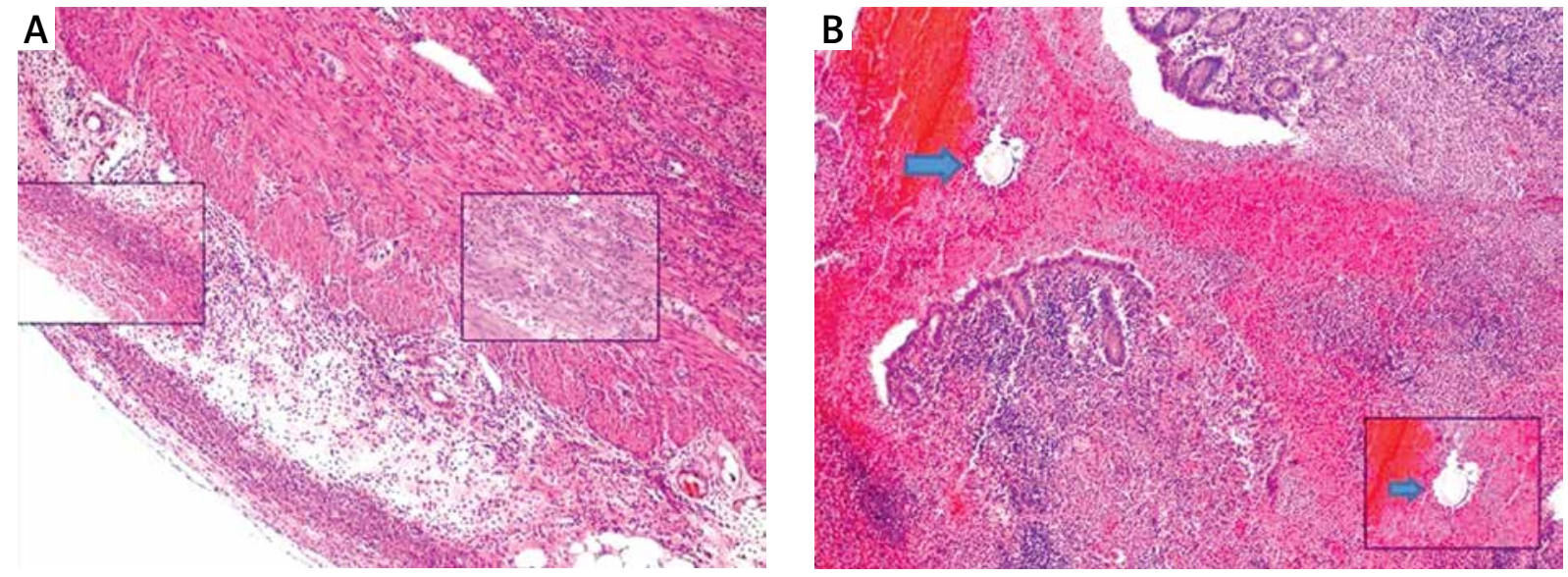

Figure 1. Case 1. A - Acute appendicitis and periappendicitis (100x, inset - 200x). B - Hair follicle in the lumen (100x, arrow-hair follicle)
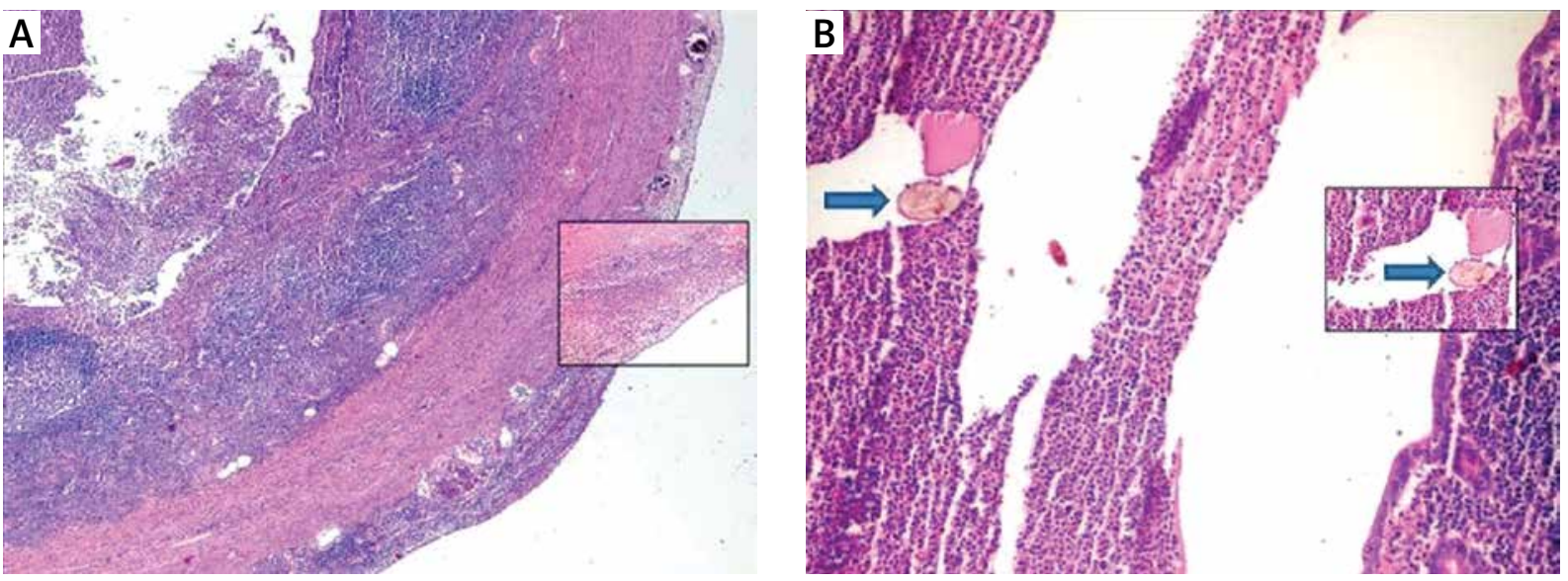

Figure 2. Case 2. A - Acute transmural appendicitis (100x, inset - 200x). B - Hair follicle in the lumen (100x, arrow-hair follicle)

materials, drill bits, toothbrush hairs, toothpicks or condom fragments [2]. Several cases of bezoar formation have been reported in the literature [3-7]. Trichobezoar formation in the gastrointestinal tract is mostly seen in women with psychotic disorders or children with mental retardation $[4,6,8]$. Trichobezoars are usually seen in the stomach but may also be seen rarely in the bowels [8].

Appendicitis due to a foreign body obstruction may be asymptomatic in some cases while causing various symptoms such as diarrhoea, vomiting, or abdominal pain without diarrhoea or vomiting in others. Increased WBC counts and CRP levels may be seen depending on the severity of the inflammation. Computerised tomography may be needed to confirm the diagnosis and to estimate the size of the trichobezoar [4].

Our patients had clinically acute appendicitis. The hair follicle in the lumen that was revealed by the pathological examination caught our attention due to its rarity. Neither of the patients had hair loss or trichotillomania in their history. Their psychiatric examinations revealed no significant psychotic disorders.

In conclusion, the possibility of foreign body obstruction and the presence of a gastrointestinal trichobezoar should be kept in mind in patients with appendicitis. Additionally, in patients suspected to have trichobezoar, who undergo appendectomy, care should be exercised to not leave hair formation in the body, to avoid re-surgery, and psychiatric consultation is required after the operation.

\section{Conflict of interest}

The authors declare no conflict of interest.

\section{References}

1. Hazer B, Dandin Ö, Karakaş DÖ. A rare cause of acute appendicitis: an ingested foreign body. Ulus Travma Acil Cerr Derg 2013; 19: 570-2. 
2. Sar S, Mahawar KK, Marsh R, et al. Recurrent appendicitis following successful management of an appendicular mass in association with a foreign body: a case report. Cases J 2009; 2: 7776.

3. Attard GJ. Trichobezoar - an unusual cause of acute appendicitis. J R Army Med Corps 1983; 129: 117.

4. Al Wadan AH, Al Kaff H, Al Senabani J, et al. Rapunzel syndrome trichobezoar in a 7-year-old-girl: a case report. Cases 2008; 1: 205.

5. Kochar AS. Acute appendicitis associated with a trichobezoar. JAMA 1984; 252: 1681-2.

6. Dogra S, Yadav YK, Sharma U, et al. Rapunzel syndrome causing appendicitis in an 8-year-old girl. Int J Trichol 2012; 4: 278-9.

7. Dehghan A, Moaddab AH, Mozafarpour S. An unusual localization of trichobezoar in the appendix. Turk J Gastroenterol 2011; 22: 357-8.

8. Alsafwah S, Alzein M. Small bowel obstruction due to trichobezoar: role of upper endoscopy in diagnosis. Gastrointest Endosc 2001; 52: 784-6.

Received: 12.09.2016

Accepted: 18.11 .2016 